Itinéraires Itinéraires

Littérature, textes, cultures

2011-1| 2011

Les Mémoires, une question de genre?

\title{
Pour une poétique queer de Rimbaud
}

\section{Max Kramer}

\section{OpenEdition}

\section{Journals}

Édition électronique

URL : http://journals.openedition.org/itineraires/1658

DOI : $10.4000 /$ itineraires. 1658

ISSN : 2427-920X

Éditeur

Pléiade

\section{Édition imprimée}

Date de publication : 1 avril 2011

Pagination : 123-136

ISBN : 978-2-296-13692-2

ISSN : 2100-1340

\section{Référence électronique}

Max Kramer, « Pour une poétique queer de Rimbaud », Itinéraires [En ligne], 2011-1 | 2011, mis en ligne le 01 avril 2011, consulté le 26 juin 2020. URL : http://journals.openedition.org/itineraires/1658 ; DOI : https://doi.org/10.4000/itineraires. 1658

\section{(ब) $(\Theta \Theta$}

Itinéraires est mis à disposition selon les termes de la licence Creative Commons Attribution - Pas d'Utilisation Commerciale - Pas de Modification 4.0 International. 


\title{
Pour une poétique queer de Rimbaud
}

\begin{abstract}
Sexology and sexual taboos had an enormous influence on Rimbaud's poetry. Rimbaud devised a number of queer strategies - particularly queer metaphor - in order to comment on his sexuality obliquely in an increasingly homophobic age. This essay argues that queer studies should pay more, and more systematic, attention to these strategies and put an end to a long-standing heteronormative uneasiness characteristic of the reception of Rimbaud's work.
\end{abstract}

Keywords : Rimbaud, sexology, queer studies, heteronormativity, metaphor Mots clés : Rimbaud, sexologie, études queer, hétéronormativité, métaphore

L'aspect tabou des représentations que l'on trouve dans l'œuvre rimbaldienne accompagne aussi leur réception critique. Un sujet sensible à aborder en poésie est resté sensible comme objet d'enquête critique dans les décennies qui ont suivi. Pendant longtemps, la critique est restée prisonnière de la même culture hétéronormative qui avait informé les poèmes eux-mêmes. Il est extrêmement rare, avant la lente ascension de la politique identitaire et le développement des études des minorités depuis les années 1970, de trouver une critique qui débatte directement les aspects queer dans la poésie rimbaldienne. Ces aspects étaient souvent considérés comme non pertinents ou, tout au plus, discutés comme sous-rubrique.

Cette réticence connaît aussi une autre cause : de façon quelque peu perverse, la plupart des textes fondateurs des études queer consacrés à la période moderniste ont surtout porté sur des œuvres en prose et ont négligé pour une grande part les textes poétiques, notamment non anglophones. Le Between Men ${ }^{1}$ d'Eve Kosofsky Sedgwick traite, certes, de Tennyson et de Shakespeare, mais n'aborde l'œuvre de Whitman qu'indirectement par le biais d'une discussion féministe de son influence sur divers écrivains

1. Eve Kosofsky Sedgwick, Between Men. English Literature and Male Homosocial Desire, New York, Columbia UP, 1985. 
anglais. Dans Épistémologie du placard ${ }^{2}$, Sedgwick concentre entièrement son attention sur des prosateurs. Il en est de même pour Jonathan Dollimore dans Sexual Dissidence ${ }^{3}$, James Creech dans Closet Writing/Gay Reading ${ }^{4}$ et Lee Edelman dans Homographesis ${ }^{5}$, même si ce dernier aborde Wallace Stevens dans un chapitre sur l'efféminement de la littérature. Somme toute, l'œuvre de Rimbaud n'a pas autant bénéficié que d'autres du développement de ce type d'études. Le critique cherche en vain des monographies qui s'attaquent de front aux questions queer dans la poésie de Rimbaud, c'est-à-dire, des monographies qui ne traitent pas ces questions comme des ajouts de dernière minute.

C'est également un problème dans la critique rimbaldienne : les multiples manifestations de la notion de queer que l'on peut distinguer dans la poésie de Rimbaud n'ont pas été examinées comme un tout interdépendant, comme parties d'une même question fondamentale. Au contraire, les critiques ont souvent préféré traiter chaque textualité sexuelle comme un phénomène séparé et ont, par exemple, traité ce que l'on a choisi d'appeler obscénité dans le « Sonnet du trou du cul » et dans « Parade » - Antoine Fongaro $^{6}$ y interprète l'expression « ressources dangereuses » dans le poème comme le pénis et « prendre du dos » comme une référence au coït anal - comme étant un phénomène sans relation, par exemple, avec le viol, évoqué dans « Le Cour du pitre ». Bien sûr, il faut reconnaître qu'il existe des distinctions de taille entre ces phénomènes. Toutefois, au moyen du concept queer, terme générique qui englobe toutes les constellations du genre et de la sexualité hors normes, il est aujourd'hui possible d'analyser les corrélations entre des manifestations sexuelles en apparence très différentes. Qui plus est, il semble désormais envisageable de combiner en un seul concept, d'une part, les théories tirées des études de genre (gender studies) et celles des études queer (queer studies) avec, d'autre part, les théories sur la référence métaphorique, stratégie majeure dans l'œuvre de Rimbaud.

Il faut, en même temps, constater que les recherches rimbaldiennes ont commencé assez tôt à reconnaître les éléments homosexuels, sinon

2. Eve Kosofsky Sedgwick, Épistémologie du placard, trad. Maxime Cervulle, Paris, Amsterdam, 2008 [1990].

3. Jonathan Dollimore, Sexual Dissidence, New York, Oxford UP, 1991.

4. James Creech, Closet Writing / Gay Reading: The Case of Melville's Pierre, Chicago, University of Chicago Press, 1993.

5. Lee Edelman, Homographesis, New York, Routledge, 1994. Pour une traduction en français du premier chapitre de cet ouvrage (« Homographèse : identité corporelle et différence sexuelle ») voir Marie-Anne Paveau et Pierre Zoberman (dir.), Corpographèses : Corps écrits, corps inscrits, Itinéraires. LTC, 2009, 1, p. 133-160.

6. Antoine Fongaro, De la lettre à l'esprit : pour lire "Illuminations », Paris, Champion, coll. « Colloques, congrès et conférences sur l'Époque moderne et contemporaine », 2004, p. 132-139, p. 151-160. 
dans l'œuvre du poète, au moins dans sa biographie. L'exemple le plus marquant en est le Rimbaud vivant de Robert Goffin, publié en 1937, qui inclut la phrase programmatique hardie : « oser appeler un chat un chat et Rimbaud... un homosexuel ${ }^{7}$ ». Bien sûr, la lecture de Goffin est entièrement vieillie de par son biographisme, mais elle s'illustre par le fait qu'elle considère déjà l'homosexualité comme une « clef précieuse ${ }^{8}$ " pour analyser la poésie de Rimbaud. De surcroît, elle indique que des poèmes comme « Parade », «Antique » et « Bonne pensée du matin » méritent qu'on leur prête plus d'attention. Ce sont des poèmes qui poseront encore problème au niveau de leur cercle herméneutique des décennies après.

\section{Le malaise hétéronormatif de la critique}

Après la Seconde Guerre mondiale, expliquer les éléments queer dans la poésie rimbaldienne devient entièrement plus banal. Le fait que Rimbaud était connu de son vivant comme un enfant terrible rendait cet affranchissement bien plus aisé. Néanmoins, les analyses faites jusque dans les années 1960 et 1970, tout en laissant entendre le contenu allosexuel ${ }^{9}$ probable d'une grande partie de la poésie de Rimbaud, ne vont pas jusqu'à cracher le morceau. Quoique typique d'une approche formaliste de la littérature - alors au goût du jour - cela signale, ne serait-ce qu'en partie, un malaise hétéronormatif face au sujet. Un exemple de cette tendance est le livre de John Porter Houston, The Design of Rimbaud's Poetry ${ }^{10}$, publié en 1963. L'auteur traite, pour la première fois, « Le Cour du pitre » en termes de viol homosexuel et interprète « Délires I » comme une « métaphore sexuelle blasphématoire ${ }^{11} »$. Son jugement final est toutefois immédiatement accompagné d'un sauf-conduit : " Qu'il fasse allusion au triomphe de Sodome ou à une nouvelle ère d'amour universel reste à débattre ${ }^{12}$. » De même, dans son article, "Rimbaud's Images immondes ${ }^{13}$ », Edward J. Ahearn examine les composantes excrémentielles dans la poésie de Rimbaud sans se hasarder à inclure le « Sonnet du trou du cul » dans son étude, ce qui aurait probablement révélé que l'excrémentiel est directement lié à l'homosexualité dans la création rimbaldienne. Similairement, Antoine Fongaro,

7. Robert Goffin, Rimbaud vivant, Paris, Corrêa, 1937, p. 87.

8. Ibid., p. 151.

9. Les termes allosexuel et altersexuel et leurs variantes morphologiques seront utilisés comme des synonymes de queer. Ils sont courants surtout au Canada et ont l'avantage de s'adapter bien mieux au génie de la langue française, en particulier, quand il s'agit de rendre la notion de queerness (allosexualitélaltersexualité). Allosexuel fait, bien évidemment, écho à allophone, concept important au Canada.

10. John Porter Houston, The Design of Rimbaud's Poetry, New Haven, Yale UP, 1963.

11. Ibid., p. 171.

12. Ibid., p. 173.

13. Edward J. Ahearn, « Rimbaud's Images immondes », The French Review, vol. 40, n 4, 1967, p. 505-517. 
dans un très bref article de 1975, « Obscène Rimbaud ${ }^{14}$ », fait de la relation homosexuelle réelle de Rimbaud avec Verlaine une prémisse de son analyse de « Bonne pensée du matin » mais ne fait référence à la question en jeu, le coït anal, qu'à la manière d'un secret d'alcôve. Ce n'est pas un hasard si Fongaro ne clarifie pas sa lecture probante de l'expression « Dont l'âme est en couronne » avant 1984. C'est alors qu'il mentionne, dans une suite à son article, « Obscène Rimbaud 3 ou "l'âme en couronne" ", l'intertexte crucial : « on s'encule en couronne ${ }^{15}$ ». En guise de dernier exemple pour montrer à quel point la recherche rimbaldienne était bégueule, on citera un article de Mario Richter qui fait référence aux constatations de Fongaro mais qui s'abstient même de citer directement le « on s'encule en couronne » et parle plutôt d'un « couplet très grossier » avant de juger le « Sonnet du trou du cul » « très vulgaire ${ }^{16}$ ». Ainsi la critique littéraire se réduisit à ne produire que des jugements de valeur, incapable ou de concevoir ou de s'imaginer pourquoi Rimbaud, pourtant un artiste avéré, aurait pu vouloir s'exprimer de la façon qu'il a choisie.

Une autre façon d'éviter la sexualité non normative dans la poésie de Rimbaud et donc l'agentivité humaine ou l'histoire matérielle, comme le font de nombreuses études, est de bel et bien traiter les éléments risqués mais en les incorporant à un programme supposé supérieur et pas nécessairement sexuel. Ainsi, Shoshana Felman, dans « Tu as bien fait de partir, Arthur Rimbaud » met en avant la question du viol dans « Le Cœur du pitre » mais seulement pour interpréter la «fantasmatique du viol» comme synonyme de « sujet violé» et comme une élaboration de la question du « Je est un autre ${ }^{17} \gg$ dans la poésie de Rimbaud, et partant de là pour discuter le problème de l'altérité isolément en évacuant l'aspect queer, qui, pourtant, est une sous-catégorie importante de cette altérité, voire son moteur principal. «Une complication de texte » de Tzvetan Todorov est peut-être l'exemple le plus extrême de cette école quand on arrive à la conclusion que Rimbaud avait conçu des poèmes tels que " Conte », " Génie » ou encore «Antique »- qui font tous partie des Illuminations et qui traitent de la sexualité déviante - pour qu'ils ne puissent pas être compris. Le principal but des Illuminations serait la non-compréhension : « c'est en voulant restituer le sens de ces textes que l'exégète les en prive - car leur sens, paradoxe inverse, est de n'en point avoir ${ }^{18} »$. En voulant, dans un geste suprême,

14. Antoine Fongaro, « Obscène Rimbaud », Studi francesi, ${ }^{\circ} 19,1975$, p. 94-95.

15. Antoine Fongaro, " Obscène Rimbaud 3 ou "l'âme en couronne" ", Parade Sauvage, $\mathrm{n}^{\circ} 1,1984$, p. $40-42$, p. 41.

16. Mario Richter, «Une "étude" : “À quatre heures du matin, l'été" », Parade Sauvage, $\mathrm{n}^{\circ} 2,1990$, p. 38-51, p. 44.

17. Shoshana Felman, «Poésie et modernité : "Tu as bien fait de partir, Arthur Rimbaud" », Littérature, $\mathrm{n}^{\circ} 11,1973$, p. 3-21, p. 9.

18. Tzvetan Todorov, « Une complication de texte : les Illuminations », Poétique, $\mathrm{n}^{\circ} 34$, 1978, p. 241-253, p. 252. 
allonger une estocade à toute exégèse (trop terre-à-terre), Todorov lui aussi, fait preuve d'un aveuglement hétéronormatif.

C'est seulement à partir de la fin des années 1970 que les critiques commencent à analyser plus librement les facettes sexuellement non normatives de l'œuvre de Rimbaud. L'essai de Gérald Schaeffer, « Poèmes de la révolte et de la dérision ${ }^{19}$ » a le mérite d'être le premier à explorer en profondeur certaines stratégies queer dans la poésie de Rimbaud tout en nommant aussi explicitement les possibles activités sexuelles dans des poèmes comme « Le Cœur du pitre » et « Mes petites amoureuses ». De la même façon, «Le sacré-cœur volé du poète ${ }^{20}$ » de Steve Murphy examine minutieusement les représentations sexuelles dans la poésie rimbaldienne. Quelques années plus tard, son excellent Le Premier Rimbaud ${ }^{21}$ présente diverses lectures novatrices des poèmes queer de Rimbaud, dont « Vénus Anadyomène ». Toutefois, un peu comme c'était le cas pour Shoshana Felman dans les années 1970, les exégèses de Murphy ne sont pas centrées sur les questions queer elles-mêmes mais s'intéressent plus au fait que ces questions sont indicatives d'exploitation sexuelle, c'est-à-dire qu'il les lit comme une allégorie idéologique. En d'autres termes, malgré le fait qu'elles nomment explicitement la sexualité déviante, ces lectures n'entrent pas dans la problématique queer. Elles négligent de penser que la non-normativité de la sexualité représentée est en elle-même signification et non simplement vecteur de signification. C'est encore plus le cas pour Michael Riffaterre, qui offre une lecture de "Vénus Anadyomène » et qui lie - peut-être inconsciemment - la dimension queer de Rimbaud au concept de l'inversion, mais purement dans son sens esthétique, au sens d'une « beauté peinte a contrario » qui ne correspondrait pas à la beauté canonique mais qui « laiss[erait] dans le texte un avers intolérable, le dégoût ${ }^{22} »$. Riffaterre ne va pas plus loin et s'abstient de prendre en compte ce qui aurait pu informer cette inversion poétique, à savoir l'équation universelle homosexualité = inversion (au sens sexuel) à l'époque de Rimbaud.

Puis, les choses évoluent peu à peu. En 1985, Christiane Morinet approfondit la question de 1' « inversion des marques de genre ${ }^{23} »$, et Danielle Bandelier le fait aussi dans Se dire et se taire ${ }^{24}$ (paru en 1988),

19. Gérald Schaeffer, « Poèmes de la révolte et de la dérision », dans Yves Bonnefoy et Marc Eigeldinger (dir.), Études sur les « Poésies » de Rimbaud, Neuchâtel, La Baconnière, 1979, p. 81-132.

20. Steve Murphy, «Le sacré-cœur volé du poète », dans André Guyaux (dir.), Lectures de Rimbaud, Revue de l'Université de Bruxelles, $\mathrm{n}^{\circ}$ 1-2, 1982, p. 27-45.

21. Steve Murphy, Le Premier Rimbaud, Lyon, Presses Universitaires de Lyon, 1990.

22. Michael Riffaterre, «Rimbaud intertextuel», Parade Sauvage, n² 2, 1987, p. 93-106, p. 105.

23. Christiane Morinet, «Sur l'Époux infernal d'A. Rimbaud», dans Alain Borer, JeanPaul Corsetti et Steve Murphy (dir.), Rimbaud Multiple : Colloque de Cerisy, Paris, Bedou/ Touzot, 1985, p. 189-196, p. 190.

24. Danielle Bandelier, Se dire et se taire. Lecture d'Une saison en enfer d'Arthur Rimbaud, 
qui analyse « Délires I » relativement à un « brouillage sexuel » et suggère d'envisager la « réflexivité » comme sa fonction fondamentale. Finalement, Duplicités de Rimbaud d'André Guyaux ${ }^{25}$ discute à fond les enjeux queer dans le poème «Antique ». Les études les plus récentes n'apportent pas beaucoup de nouveau. " Figures féminines dans l'œuvre de Rimbaud » de Laura J. Poulosky ${ }^{26}$ offre une lecture féministe de «Délires I » et établit un rapport entre l'homosexualité et la misogynie. Rimbaud sans occultisme $^{27}$ et Éclats de la violence ${ }^{28}$ de Pierre Brunel analysent diverses connotations queer dans les poèmes avec une érudition incontestable et donnent un aperçu très vaste de toutes les recherches qui ont été faites. Ces analyses sont toutefois l'œuvre d'une autre génération dans leur traitement du sujet. Ce n'est qu'avec la thèse de Merrill Cole, The Erotics of Masculine Demise: Homosexual Sacrifice in Modernist Poetry ${ }^{29}$, qu'une lecture queer de l'œuvre de Rimbaud, ou du moins d'une partie de l'œuvre, a été tentée. Cole examine la manière dont l'" affect homoérotique rend inadéquates les conventions de la mimésis ${ }^{30} »$, mais il manque encore des contributions queer plus variées.

\section{Les stratégies queer rimbaldiennes}

Ce qu'il faut reconnaître dans l'œuvre de Rimbaud, c'est que les diverses formes d'altersexualité qui y sont évoquées sont en fait le produit d'une préoccupation indivisible chez le poète que l'on pourrait alors utilement mettre en rapport avec sa sexualité personnelle. Il n'est donc pas utile d'analyser les multiples stratégies queer comme des éléments discrets et hétérogènes. Ne pas voir des liens entre « Délires I » (poème dans lequel l'inversion des genres constitue la pierre angulaire), « Parade » (qui introduit le lecteur à la vision queer d'un numéro de cirque ou d'un spectacle de foire et qui joue avec la notion du placard et le dévoilement de l'identité allosexuelle) et le « Sonnet du trou du cul» ou encore « Le Cœur du pitre » et ne pas reconnaître une hantise et un travail queer communs derrière ces représentations revient à prendre Rimbaud pour un plaisantin distrait dans l'œuvre de qui « l'incohérence harmonieuse » serait le principe

Neuchâtel, La Baconnière, 1988, p. 35.

25. André Guyaux, Duplicités de Rimbaud, Paris-Genève, Champion-Slatkine, 1991.

26. Laura J. Poulosky, « Figures féminines dans l'œuvre de Rimbaud : représentantes du pouvoir, de la soumission et de la camaraderie », dans Paul Perron et Sergio Villani (dir.), Lire Rimbaud, Toronto, Canadian Scholars' Press, 2000.

27. Pierre Brunel, Rimbaud sans occultisme, Fasano, Schena, 2000.

28. Pierre Brunel, Éclats de la violence. Pour une lecture comparatiste des Illuminations d'Arthur Rimbaud, Paris, Corti, 2004.

29. Merill Coll, The Erotics of Masculine Demise: Homosexual Sacrifice in Modernist Poetry, Thèse de doctorat, Université de Washington, 1999. Les traductions des textes anglais sont de Max Kramer.

30. Ibid., résumé. 
même - formule forgée par Valéry - ou bien l'illisibilité -Todorov à propos des Illuminations - idées que, pourtant, les critiques mentionnés ci-dessus excluent du simple fait de leur engagement dans l'exégèse de l'œuvre de Rimbaud.

Les diverses stratégies queer rimbaldiennes rendaient possibles deux choses. D'une part, elles permettaient à l'auteur, dans un mouvement épistémique, de s'attaquer aux questions sexuelles qu'il n'avait probablement pas résolues pour lui-même. D'autre part, elles permettaient d'éluder les tabous moraux, les restrictions d'ordre judiciaire ainsi que la pathologisation qui frappait alors de plein fouet la sexualité de même sexe. Bref, elles permettaient à Rimbaud d'exprimer une réalité singulière, de parler des pratiques et des identités que la société du Second Empire et de la Troisième République comptait voir rester sur la touche.

Des lectures récentes ont attiré l'attention sur les non-dits sexuels dans la poésie rimbaldienne, et de nombreux théoriciens des études queer et de genre ont analysé la sexualisation de l'identité dans l'Europe de la fin du $\mathrm{XIX}^{\mathrm{e}}$ siècle. Ce qui fait défaut, ce sont des études qui explorent systématiquement l'intersection de ces deux démarches en examinant la manière dont le discours scientifique sur la sexualité se traduit chez Rimbaud en une poésie hermétique qui voile et exprime à la fois le sens sexuel par le biais de l'indirection. À titre d'exemple, on peut citer ici le très court poème « $\mathrm{H} »$, avec ses références à la « mécanique érotique » ou bien l'« ardente hygiène des races » et sa célèbre interjection finale : «terrible frisson des amours novices ». Mais il y a aussi « Les Remembrances du vieillard idiot» avec ses allusions aux " jeunes crimes » ou "Les Poètes de sept ans » qui contient de multiples évocations d'une sexualité considérée comme déviante, la masturbation entre autres (« les deux poings à l'aisne »). Cela suppose que le discours poétique rimbaldien a une fonction absolument référentielle, et c'est pourquoi il faut rattacher les théories contemporaines de la poétique à l'étude du genre, du sexe biologique et du désir sexuel.

\section{Une double lignée de médecins et de poètes}

Dans le chapitre de l'Histoire de la sexualité qui traite de l'hypothèse répressive, Michel Foucault conteste l'idée que, depuis le XVIII' siècle, il y aurait eu répression du discours sur la sexualité. Il maintient qu'au contraire, à l'époque, loin d'être muettes sur le sujet, les sociétés occidentales n'ont cessé, en fait, de tenir des discours sur le sexe. Les recherches rimbaldiennes profiteraient de la participation directe à l'étude de ce phénomène, car elles montreraient que l'expression poétique équivaut à un discours additionnel et distinct, indépendant des discours de type scientifique, confessionnel, psychanalytique et romanesque.

Foucault soutient également que l'identité sociale est devenue graduellement plus sexualisée pendant cette même période historique. 
À l'époque, on imaginait communément les personnes qui avaient des pratiques homosexuelles comme des membres d'une espèce particulière. Elles n'étaient plus simplement les auteurs d'actes sexuels déviants; on les concevait désormais comme des invertis ou homosexuels marqués par une androgynie intérieure et, logiquement, par une anomalie identitaire. Rimbaud, dont la sexualité dissidente (homosexualité ou bisexualité) comme élément biographique est incontestée, a grandi dans cet univers discursif vulgarisé par les revues érotiques - « Il s'aidait / De journaux illustrés où, rouge, il regardait / Des Espagnoles rire et des Italiennes » ( «Les Poètes de sept ans ») - et sa poésie émane de cette réalité sociale. Rimbaud, membre d'une espèce sexuelle nouvellement concoctée dans les sciences $^{31}$ et de plus en plus objet de discrimination, était probablement plus sensible que d'autres au hiatus entre la construction sociale d'une sexualité non normative et l'hétéronormativité.

Parallèlement à l'évolution des sciences et du discours sur la sexualité, la qualité de l'écriture poétique évoluait. Avec l'avènement du symbolisme, comme le remarque Catherine Détrie, la métaphore devient le définisseur de la création poétique : « le Romantisme, puis le Symbolisme et le Surréalisme auront pour visée de transformer la métaphore en un outil de connaissance créatrice permettant de signifier l'invisible et l'inconnaissable ${ }^{32} »$. Ce renouvellement de la métaphore en outil de connaissance, c'est ce qui va aider des auteurs comme Rimbaud, marginalisés dans leur vie sexuelle réelle, à transmettre la vision d'un monde distinct et étouffé par l'éthique hétéronormative. La métaphore et, en général, les procédés d'obscurcissement que l'on privilégie dans la seconde moitié du XIX ${ }^{\mathrm{e}}$ siècle sont parfaits pour la représentation nécessairement codée de la déviance sexuelle dans le climat de plus en plus homophobe du XIX ${ }^{\mathrm{e}}$ siècle. Les critiques rimbaldiens doivent interpréter cela comme la conjonction logique de deux aspects de la même préoccupation moderne : celle d'exprimer l'ineffable (social) et le soi (honteux) dans une opposition essentielle à un monde extérieur hostile.

Une raison pour laquelle l'œuvre rimbaldienne se prête particulièrement bien à une lecture queer, c'est que la création de métaphores est souvent plus fréquente dans la poésie que dans le roman ou le théâtre. Le travail de Rimbaud s'inscrit dans les principes de la modernité poétique, c'est-à-dire le rejet de la rhétorique didactique, la suggestion et les préoccupations d'idéal ou d'absolu, principes qui sont tous aidés par la métaphore. En outre, le lectorat tend à plus associer le poème à la vie réelle de son auteur qu'il ne le fait pour le roman ou la pièce de théâtre, surtout quand les mœurs ou l'identité allosexuelles se rapportent à un personnage clairement

31. Dans le cadre nécessairement limité du présent essai, il n'est pas possible de discuter cette chronologie foucaldienne ni sa remise en cause ces dernières années par les études sur les périodes plus anciennes.

32. Catherine Détrie, Du sens dans le processus métaphorique, Paris, Champion, 2001, p. 67. 
distinct du narrateur ou du protagoniste, comme Charlus et Albertine dans le cas de Proust ${ }^{33}$. En poésie, ce qui est fictif est souvent assimilé au biographique. Il est possible que ce soit précisément pour cette raison qu'Oscar Wilde a exhorté André Gide à ne plus jamais écrire Je. Il en résulte que le poète court potentiellement plus de risques que les autres auteurs quant aux signifiants interdits et est donc vraisemblablement plus porté à avoir recours à des tactiques de diversion telles que la métaphore.

L'autre raison d'être d'une lecture queer de l'œuvre rimbaldienne est que l'auteur a été témoin de l'avènement de la sexologie, la modernité poétique correspondant à peu près à l'élaboration ou à l'emploi extensif dans le domaine médical de termes clés tels qu'inversion, homosexualité, hystérie, sadisme, masochisme, perversion, masturbation et ainsi de suite. Brunel remarque à bon droit que le $\mathrm{XIX}^{\mathrm{e}}$ siècle amène une « double lignée de médecins et de poètes ${ }^{34} \gg$. Cette période s'étend approximativement jusqu'aux années menant à la Seconde Guerre mondiale et c'est en France qu'apparaît d'abord le mouvement symboliste. Il se caractérise par la co-présence de divers paradigmes de la sexualité, parmi lesquels le paradigme antique et le paradigme chrétien qui commencent à être dépassés mais qui influencent encore les mœurs. Pendant cette période, la poésie joue abondamment avec la notion du mal dans la sexualité ou avec les transgressions sexuelles - on pourrait penser aux Fleurs du mal de Baudelaire comme point de départ emblématique pour cette poésie. Cette tendance, au moins sous sa forme euro-américaine, s'éteint avec les horreurs de la Seconde Guerre mondiale et de la Shoah. Au même moment, à la suite de ces événements tragiques et de l'essor, ensuite, de la communauté gay comme acteur politique de mieux en mieux accepté dans l'ordre bourgeois, il y a moins de raisons de camoufler les questions queer dans la représentation poétique. La métaphore devient alors obsolète, ou au moins n'est plus une nécessité existentielle. D'un autre côté, la métaphore queer n'était pas fréquente dans la poésie avant la seconde moitié du XIX ${ }^{e}$ siècle, la sexualité déviante n'étant pas encore devenue une question sociale de premier ordre que diverses autorités, pédagogiques, médicales, etc., essayaient de contrôler. Auparavant, John Wilmot, Crébillon, Laclos, Casanova, Sade et autres auteurs s'étaient, bien sûr, déjà penchés sur des questions d'ordre sexuel. Cependant, les œuvres de la plupart de ces auteurs, étaient en prose et s'inscrivaient dans la tradition libertine. Cette marque d'une aristocratie qui jouit d'une certaine liberté sexuelle et qui n'est pas contrainte par la morale, contrairement au reste de la société, expire essentiellement avec la Révolution française. Ces valeurs sont ainsi liées à

33. On notera cependant que, dans le cas de Proust, la tentation est forte d'assimiler l'auteur et le narrateur, et de vouloir prendre le narrateur/Proust en défaut, comme l'atteste encore le Chambre 43. Un lapsus de Proust, de M. Lavagetto (trad. A. Pasquali, Paris, Belin, 1996 [1993]).

34. Pierre Brunel, Rimbaud sans occultisme, op. cit., p. 217. 
un ordre qui disparaît, à un effort ultime pour s'accrocher à une liberté, bien que parfois, comme c'est le cas pour Sade, elle soit très problématique.

On doit s'attendre à ce que la poésie queer ait été surtout l'œuvre d'auteurs qui étaient aux prises avec une dichotomie entre sexualité normale et sexualité anormale. De surcroît, 1'homosexualité masculine offrait la plus forte tension entre ce qui était sexuellement acceptable et ce qui ne l'était pas. L'idéologie sexiste masculine présumait que le renversement du paradigme des genres (l'inversion) était intrinsèque aux relations sexuelles entre hommes - avec la conviction sous-jacente qu'un des deux partenaires s'identifiait au genre féminin - et le considérait donc comme dégradant pour un homme puisque celui-ci renonçait à son rôle d'homme, et donc à son statut moral, social, etc., supérieur. Les hommes, et donc l'establishment littéraire, se montraient plus libéraux envers le lesbianisme, surtout quand ce n'était pas des femmes mais d'autres hommes qui le rendaient public. Assurément, les poèmes lesbiens de Baudelaire (Les Épaves) étaient censurés en France mais Baudelaire pouvait toutefois les publier en Belgique. C'est aussi ce qu'a pu faire Verlaine peu après, en ce qui concerne les six sonnets intitulés "Les Amies ». S'ajoutent à cela 1' « Erinna » de Banville, Les Chansons de Bilitis de Pierre Louÿs, ou « Anactoria », «Faustine » et « Sapphics » de Swinburne. Ces œuvres profitaient du fait que le public masculin ne les trouvait pas nécessairement incompatibles avec la sexualité normale parce qu'il les considérait comme des œuvres voyeuristes qui permettaient aux hommes hétérosexuels de découvrir la sexualité féminine. Elles les titillaient même quand elles avaient un ton moralisateur ou condamnateur. Le lesbianisme était, en définitive, moins menaçant pour l'ordre social. Les soi-disant amitiés romantiques, c'est-à-dire, les amitiés intimes entre femmes, étaient florissantes à la même époque et étaient souvent entièrement acceptées dans la culture du XIX ${ }^{\mathrm{e}}$ siècle.

Dans les limites chronologiques de l'ère moderniste, Rimbaud est le premier à exprimer un monde véritablement queer. Il est vrai que l'on pourrait aussi penser à Verlaine, qui a écrit une série de poèmes entièrement consacrés à l'homosexualité masculine et féminine. De toute façon, ces deux recueils de Verlaine, Femmes et Hombres, sont complètement explicites et donc peu métaphoriques, fait qui y est même érigé en principe : «Ne métaphorons pas, foutons ». Publiés clandestinement, ils n'ont pas eu à confronter la pensée hétéronormative, rendant inutile l'emploi d'un masquage stratégique. Rimbaud, en revanche, a élaboré une façon unique de voir la métaphore et la sexualité queer. Son œuvre reflète (positivement ou négativement) le Zeitgeist de l'époque sur ce qui alors a trait à la sexualité en France et en Europe, en particulier à la pathologisation de certaines pratiques sexuelles, qui sont communiquées par le biais du subterfuge. 


\section{La métaphore queer}

Ce qui est une tentative d'ambiguité et d'impersonnalité dans la poésie de Mallarmé, signifiant une absence - le poète se repliant derrière le texte poétique - pouvait devenir une déclaration figurative et soigneusement codée sur la sexualité, sous la plume de Rimbaud. Cette textualité sexuelle a pu engendrer de multiples analyses contradictoires, et l'a fait. Dans certaines lectures, le texte rimbaldien échappe en apparence à la doxa hétéronormative de l'époque mais, par la même occasion, défie la dénotation et toute référentialité clairement identifiable. C'est le cas, par exemple, dans «Antique » où la question de liminalité entre homme et animal (fils de Pan) se déplace vers la question de liminalité entre homme et femme, car dans le ventre de ce fils de Pan " dort le double sexe ». Le fils du protecteur des bergers et des troupeaux est devenu Hermaphrodite, le personnage mythologique bisexué. Bien que traiter le sujet de l'androgynie n'eût rien de singulier à l'époque, la représentation rimbaldienne se distingue de celle de Swinburne, de Banville ou de Gautier notamment par le fait qu'elle offre une lecture positive de l'hermaphroditisme tandis que les autres auteurs en faisaient un symbole de pathologie et de dégénérescence, comme le montre Guyaux : " Le texte de Rimbaud est au plus loin de 1" "hermaphroditisme morbide" de la fin du XIX $x^{e}$ siècle. En dépit du sujet et son succès postromantique, il ne relève pas de la littérature décadente ${ }^{35}$. » Contrairement à la doxa actuelle, Rimbaud décrit « le double sexe» comme une sorte de perfection, et cela grâce à des stratégies queer sophistiquées. Dans d'autres lectures, plus communes, le texte se conforme à l'opinion hétéronormative majoritaire sur la sexualité et est dans son ensemble considéré comme inoffensif sur le plan sexuel, comme c'est le cas avec «Voyelles » où le « Ses Yeux » final semble d'abord ne pas poser un problème particulièrement queer mais où une lecture plus attentive fait apparaître une incertitude, voire une indécidabilité, sur le genre de l'être à qui appartiennent ces yeux : renvoient-ils à une femme, à un homme, à Dieu (la représentation de l'androgynie par excellence, dans certaines cultures)? Dans cette confusion d'interprétations, la signification allosexuelle, et le poète allosexuel avec, pouvaient se cacher, échapper aux poursuites, et être universellement acceptés.

C'est ainsi que dans la poésie rimbaldienne la métaphore et la notion de queer partagent un fondement conceptuel commun. Les constructions et de la métaphore et de la sexualité de même sexe dominantes au XIX ${ }^{\mathrm{e}}$ siècle se ressemblent en ce que toutes les deux représentent des exceptions. Pour la majorité des lecteurs de l'époque, la métaphore est queer. La métaphore est une déviance linguistique, la sexualité homosexuelle une déviance sociale. Si celle-ci représente une récusation de la représentation mimétique d'une réalité laide et souvent haïe de la fin de siècle industrialisée et matérialiste, celle-là conteste la notion normative de l'expression sexuelle comme étant 
un désir sexuel pour le sexe opposé et la reproduction. Il est fort probable, étant donné le risque que comporte une telle entreprise, que les poètes modernistes comme Rimbaud qui voulaient décrire la déviance sexuelle dans un monde de normes sexuelles institutionnalisées ont eu aussi recours à la déviance linguistique, d'où la métaphore. Cette stratégie avait des chances de réussir, particulièrement à une époque où, pour la plupart des lecteurs, la métaphore est encore une citation littéraire décorative et gratuite exprimant quelque chose de non essentiel à la compréhension photoréaliste du poème. Cette stratégie rimbaldienne pourrait se résumer dans l'expression métaphore queer...

Cette métaphore queer ou allosexuelle se définit chez Rimbaud comme une métaphore vive, selon l'expression de Paul Ricœur, laquelle ne se réduit pas à la combinaison d'unités lexicales grand public préexistantes, mais fournit aussi un nouveau sens dissident. Ici cependant, la signification de la métaphore dépasse celle qu'en donne Ricœur, car la métaphore rejoint une parente proche, la déviance sexuelle. Chez Rimbaud il faut faire remonter la métaphore queer non pas au système langagier hétéronormatif standard officiellement contrôlé et peut-être fictif (la langue de Saussure) mais à la parole, le discours individualisé, minorisant et bien réel. La métaphore queer y est une perception idiosyncratique qui ne dépend pas de la définition fossilisée des mots du dictionnaire mais communique des sujets sexuels qui remettent en question les normes socio-sexuelles de l'époque. La métaphore est un outil contre ce qu'Adorno appelait l' " intolérance de l'ambiguïté », contre la rectitude et la franchise. Elle est une déviation perpétuelle par rapport à une norme présumée essentielle et insiste pour laisser intacte l'ambiguïté. Par le biais de cette déviation perpétuelle, elle remet en question la notion d'une présence précédant cette prétendue déviation. Par des réitérations dans de nouveaux contextes, par une performativité butlérienne, la métaphore queer démasque à la fois l'illusion du sens premier des mots et la notion d'identité sexuelle fixe. Participant à une chaîne de sens toujours différant et différés - la différance derridienne - la métaphore queer fait voler en éclat le semblant d'autorité et de normalité socio-sexuelle qui entoure le langage littéral et la sexualité normative. Révélant que le littéral et le normatif sont eux-mêmes des substituts provisoires, mimétiques, la métaphore queer les montre comme des autorités toujours différées au sein de régimes réglementaires, c'est-à-dire, semblables au discours figuratif et à la sexualité déviante. La métaphore queer fait valoir précisément l'expérience qui est marginale et restreinte par les tabous sexuels. La poésie rimbaldienne rend visible la déviance sexuelle mais la reconstruit à l'intérieur des frontières de la textualité métaphorique. Il est certain que Rimbaud a employé d'autres tactiques rhétoriques d'obscurcissement mais, plus que les autres tactiques, la métaphore reste chez lui particulièrement pertinente pour la représentation de la déviance sexuelle parce qu'elle-même était conçue comme exemple de déviance dans une société hétéronormative. 
Cette poiesis métaphorique a un double objet chez Rimbaud : elle voile dans sa poésie les réalités sexuellement déviantes comme le désir homosexuel et le coït anal dans le « Sonnet du trou du cul », par exemple, ou le viol par fellation dans « Le Cœur du pitre », et ce faisant, exprime paradoxalement ces réalités. Même si ce n'est que de la façon la plus indirecte, l'expérience de la déviance trouve ses mots. Cela fait de la métaphore queer une fonction du placard. La sexualité queer peut parler en son propre nom et la métaphore exprime cette sexualité pour les lecteurs sensibles à ses allusions. En même temps, elle protège le poète d'un groupe beaucoup plus important des lecteurs qui risqueraient probablement de désapprouver le contenu altersexuel, ou bien, qui ne parviendraient pas à se mettre à la place du je lyrique. Les métaphores rimbaldiennes dissimulent tout en révélant. Elles facilitent la peinture de ce qui est proscrit, désapprouvé ou rejeté dans le silence. En outre, la métaphore queer a une qualité épistémique. Elle permet à Rimbaud de faire face à quelque chose qu'il n'avait pas résolu pour lui-même et qu'il vivait comme un dilemme psychologique et social voir la crise de sujet (queer) décrite dans « Délires I », qui s'exprime dans la phrase « Je n'aime pas les femmes » et son pendant qui le suit immédiatement : «L'amour est à réinventer ».

\section{La projection queer}

C'est pourquoi dans le cas de Rimbaud, tout en prêtant attention surtout aux poèmes, la discussion devra tenir compte du fait biographique, à savoir qu'il a rédigé la plupart de ses poèmes de la position marginalisée de l'allosexualité. Le fait biographique de son désir homosexuel, c'est le fondement du postulat qu'il était très sensible à la marginalisation sexuelle ainsi qu'à la différence en général. Cela amène au corollaire que cette sensibilisation pénètre son œuvre et la conditionne. De la même façon, les analyses devront rejeter la notion d'une instance autoriale neutre pour la personnalité du critique. C'est ce que James Creech a appelé une « position désincorporée ${ }^{36} »$. Quand on a affaire à la textualité queer, une telle approche est la plupart du temps une position hétéronormative. Au lieu de cela, il faudrait une approche critique qui reconnaît sa position subjective, et adopte une projection altersexuelle, un « fantasme projectif », pour offrir une exégèse qui s'apparente à la « lecture $\operatorname{camp}^{37}$ » de Creech. Une telle approche proposerait comme hypothèse qu'une grande partie des écrits rimbaldiens ont été rédigés dans une perspective queer, d'où la nécessité d'une poétique queer. Rimbaud a bien sûr dû et pu conventionnaliser le désir et l'identité altersexuels et les élever à un niveau universel mais c'est au critique qu'il incombe d'aller au-delà de ces surfaces artificieuses et d'analyser les circonstances minorées et les rapports de pouvoir précis d'où ils se sont matérialisés. La projection queer

36. James Creech, op. cit., p. 42.

37. Ibid., p. 37. 
est une négociation. Elle diffère du biographisme, car elle ne projette pas directement des détails biographiques sur le texte. Elle se consacre plutôt à la logique du texte et à sa relation énigmatique avec le discours social. Par conséquent, elle tient compte des circonstances culturelles et de la vie personnelle de l'auteur. La projection queer est toutefois un point de départ et non pas un jugement final. Ceci ne réduit en rien la valeur des œuvres poétiques parce que « l'universalité littéraire [...] a toujours émergé d'une particularité incorporée déclarée représentative ${ }^{38} \gg$. Cela ne diminue non plus en rien la validité des conclusions du critique. L'agentivité, par définition, s'imbibe de la contingence culturelle, comme le fait tout auteur, et est donc limitée ou partiale. Bien évidemment la projection queer n'est absolument pas la seule manière d'aborder la création poétique de Rimbaud. Néanmoins, toute discussion tangentielle aux questions de genre, de sexe, d'amour, de désir, d'affection, de sensualité ou d'amitié dans l'œuvre rimbaldienne sera viciée dès l'origine si elle ne tient pas compte de leur dimension queer.

Les futures recherches rimbaldiennes devront inclure des études sur le rapport entre l'œuvre de Rimbaud et les discours sur la sexualité au $\mathrm{XIX}^{\mathrm{e}}$ siècle. Cette période de changements politiques et économiques importants a fortement remis en cause les représentations liées à la sexualité, ainsi qu'en témoigne la poésie rimbaldienne. À partir des discours existants sur la sexualité, il faudra tenter d'élucider les métaphores sexuelles à l'œuvre dans cette poésie. À l'aide des théories de la métaphore de Ricœur, on pourra montrer que c'est grâce aux métaphores vives exprimant une subjectivité et un désir sexuels instables que Rimbaud a contribué à ce qu'on pourrait nommer la fin de la vérité ontologique sur la sexualité. En effet, les métaphores ne font pas que refléter un écart lexical ou une interaction prédicative : elles attaquent le rapport entre une réalité structurée d'une manière hétérosexuelle et un désir homosexuel refoulé. Ce que des conceptions rhétoriques et linguistiques identifient comme un écart est chez Rimbaud le lieu d'un conflit social profond à propos des catégories fondamentales de la réalité. La révolution sexuelle et tropologique entamée par Rimbaud produit une textualité queer qui ne se réfère plus à la réalité hétéronormative mais à une réalité tout de même. L'une des principales tâches à mener à bien pour la critique de l'œuvre rimbaldienne est d'identifier les procédés modernes spécifiques de création de métaphores allosexuelles et de montrer que la métaphoricité doit prendre en charge une dimension historique, car c'est le rejet des limites traditionnellement attachées à l'emploi de la métaphore qui permit à Rimbaud de dépasser les limites de la représentation queer.

Max Kramer

Université de Saskatchewan

38. James Creech, op. cit., p. 27. 